\title{
Transforaminal interbody debridement and fusion with antibiotics impregnated bone graft to treat pyogenic discitis and vertebral osteomyelitis: A technique note with preliminary result.
}

\section{Hsiao-Kang Chang}

Department of Orthopedic Surgery,Kaohsiung Chang Gung Memorial Hospital

\section{Meng-Ling Lu}

Department of Orthopedic Surgery,Kaohsiung Chang Gung Memorial Hospital

\section{Adam M. Wegner}

OrthoCarolina, Winston-Salem,North Carolina

\section{Re-Wen Wu}

Department of Orthopedic Surgery,Kaohsiung Chang Gung Memorial Hospital

\section{Sung-Hsiung Chen}

Department of Orthopedic Surgery,Kaohsiung Chang Gung Memorial Hospital

\section{Chieh-Cheng Hsu}

Department of Orthopedic Surgery,Kaohsiung Chang Gung Memorial Hospital

\section{Hou-Tsung Chen}

Department of Orthopedic Surgery,Kaohsiung Chang Gung Memorial Hospital

Tsung-Cheng Yin ( $\nabla$ tsongchenyin@gmail.com )

Kaohsiung Chang Gung Memorial Hospital https://orcid.org/0000-0002-2468-6196

\section{Technical note}

Keywords: infectious spondylodiscitis, parenteral antibiotics, transforaminal interbody debridement and fusion, antibiotics-impregnated bone graft, pyogenic discitis and vertebral osteomyelitis

Posted Date: July 15th, 2020

DOl: https://doi.org/10.21203/rs.3.rs-41300/v1

License: (c) (1) This work is licensed under a Creative Commons Attribution 4.0 International License. Read Full License 


\section{Abstract \\ Background}

Surgical treatment of pyogenic discitis and vertebral osteomyelitis (PDVO) is indicated for neurologic deficit, spinal instability, unknown pathogen, poorly controlled infection, or intractable pain. Although the posterior-only approach has been proved a safe, effective procedure that minimizes the risks and complications of anterior or staged surgery, parenteral antibiotic treatment for 4- 6 weeks postoperatively is still necessary. We hypothesized that antibiotic-impregnated bone graft used in an all posterior approach could result in infection control and shorten the postoperative course of pyogenic discitis and vertebral osteomyelitis.

\section{Methods}

21 consecutive patients with pyogenic discitis and vertebral osteomyelitis of the lumbar or thoracic spine were treated with transforaminal interbody debridement and fusion (TIDF) with antibiotic-impregnated bone graft (AIBG) between March 2014 and January 2017. Minimum follow up was 2 years. Outcomes included visual analog scale (VAS) back pain, ASIA scale for neurological status, kyphotic angle correction, fusion status, and functional outcome using Kirkaldy-Willis criteria, and c-reactive protein (CRP) levels. CRP levels from pre-op, immediately post-op, and 1, 2, 4 \& 6 wks post operatively and the duration of treatment with postoperative IV antibiotics in our patients was compared to our previous case series in which TIDF was performed without AIBG.

\section{Results}

Surgical treatment for PDVO resulted in clinical improvement and adequate infection control. There was no difference in pre-op CRP levels between the two groups. Despite shorter post-op IV antibiotic duration (Mean $21.0 \mathrm{~d}$ vs $39.8 \mathrm{~d}$ ), the AIBG group had a stable decline in CRP levels and continued to decrease at $1,2,4$ \& 6 weeks, with significantly lower CRP levels at 6 weeks compared to bone graft without antibiotics. VAS scores improved from a mean of 7.2 to 2.3 one month postoperatively. Patients who had increased kyphotic angles had an average angle correction of $7.9^{\circ}$ at last follow-up.

\section{Conclusion}

The technique of TIDF combined with AIBG can achieve local infection control with faster reduction in $\mathrm{CRP}$, leading to shorter antibiotic duration for pyogenic discitis and vertebral osteomyelitis

\section{Background}


Pyogenic discitis and vertebral osteomyelitis (PDVO) accounts for the majority of spinal infections, which commonly involve the lumbar region [1]. Although a wide variety of pathogens have been associated with PDVO, Staphylococcus auerus remains the most predominant [2].

Treatment varies depending on infection status, clinical symptoms, and comorbidities [2]. Surgical treatment may be indicated for those who respond poorly to antibiotic treatment or those who have spinal instability, extensive bony destruction, epidural abscess, neurological impairment, or intractable pain, or those whose needle biopsies fail to identify a definite pathogen [2-6]. Because the lesions are almost always located in the vertebral body and disc space, anterior debridement with bone graft from anterior, anterolateral (transthoracic), or lateral retroperitoneal approaches with or without posterior stabilization have been strongly advocated [7-10], though anterior approaches are also associated with higher complication rates than posterior approaches [11, 12].

Today, an increasing number of studies demonstrate the effectiveness of posterior-only approaches for the treatment of PDVO [5, 13-15]. Although the posterior approach has proven to be safe and effective with minimal risk and fewer complications than anterior approaches, there remains a need for 4 to 6 weeks of postoperative IV antibiotic treatment. Transforaminal interbody debridement and fusion (TIDF) is a posterior only approach that has been reported to achieve satisfactory results, though it also requires a long duration of post-operative antibiotic treatment $[5,16]$.

Recently, use of antibiotic-impregnated bone graft (AIBG) has yielded encouraging results [17-21]. The major benefit of this technique is a high local concentration of antibiotics, greatly exceeding concentrations following systemic administration [22]. Most studies discuss the use of AIBG in the surgical treatment of orthopedic trauma or joint arthroplasty infections $[17,18,20,21]$, but few focus on the use of AIBG in spine surgery.

In this study, the effectiveness of TIDF with AIBG for PDVO is assessed, with the hypothesis that this technique would shorten the time needed for post-operative IV antibiotics as well as time to normal CRP levels.

\section{Methods}

21 consecutive PVDO patients treated with TIDF with AIBG at a single institution between March 2014 and January 2017 with minimum of 2 years follow-up were retrospectively enrolled. Clinical and radiographic data were collected for all patients with thoracic or lumbar spine infections and who presented with neurologic deficit, spine instability, or intractable pain, and whose pathogens had not been identified or whose response to antibiotics had failed. Patients with tuberculosis bacilli infection and post-spinal operation infections were excluded. The study was approved by the institutional review board (CRMH IRB No. 201801075B0).

From the medical record, demographic data, diagnosis, affected levels, placement of screws, imaging results, laboratory data, duration of antibiotic treatment, neurologic status, and functional outcomes were 
collected. All patients wore Taylor braces for at least 3 months after surgery. Patients were followed-up at two weeks, and then one, three, and six months, then annually until at least 2 years.

Choice of parenteral antibiotics was based opinion of an infectious disease specialist and culture results. Because some patients initially receiving conservative treatment, antibiotic treatment of some patients began prior to their operations. Serial C-reactive protein (CRP) levels were collected. The normal range of CRP is below $5.0 \mathrm{mg} / \mathrm{L}$. The duration of treatment using parenteral antibiotics was determined based on clinical symptoms and lab parameters. If the patient's CRP declined to below $25 \mathrm{mg} / \mathrm{L}$ and they were afebrile without any signs of infection, we discharged the patient on oral antibiotics. All patients were prescribed oral antibiotics for $2 \sim 3$ months after discharge.

The clinical outcomes assessed were VAS for back pain and the American Spinal Injury Association (ASIA) impairment scale for neurological status. In addition, local kyphotic angles of the superior and inferior endplates of the affected levels were measured on standing XR before and after surgery and at final follow-up. Fusion was assessed by two of our authors who didn't participate in the surgery on the final follow up x-ray and defined as continuous trabeculae bridging across the interface of the treated segments, whereas the presence of any radiolucent interruption at the treated segments was defined as pseudarthrosis. Functional outcomes were evaluated using the Kirkaldy-Willis classification [23]. Finally, CRP levels and duration of antibiotic administration in the AIBG patients was compared to a control group $[5,11]$ from a cohort of 28 patients receiving an identical TIDF procedure with the same indications but without AIBG.

\section{Statistical analysis}

Differences between the "non-antibiotic-impregnated control group" and the "antibiotic-impregnated group" were compared using the Mann-Whitney U test. All statistical operations were performed using SPSS version 22.0 (IBM, Armonk, NY, USA). A p-value $<0.05$ was considered significant.

\section{Operative procedures technique note}

A modified transforaminal lumbar interbody fusion (TLIF) surgical approach was performed. This single posterior approach included debridement, fusion, and pedicle screw instrumentation. A midline open approach was used keeping the posterior elements of the spine as intact as possible. The paraspinal muscles were detached subperiosteally. Unilateral facetectomy and limited laminotomy were performed (Fig. 1A), followed by radical debridement to remove abscess, infected disc material, endplates, and sequestrum from the intervertebral space by curved curettes and disc roungeurs. (Fig. 1B). The site of entry was determined based on the expected extend of debridement necessary based on symptom severity, radiographs, CT, and MRI. Posterior stability was maintained by preserving the spinous processes, supraspinous ligaments, and the contralateral facet joint if possible.

Tissue samples were analyzed for aerobic, anaerobic, tuberculous bacilli, and fungi. Residual tissue was sent for histopathologic examination. If iliac autograft was not sufficient to fill the residual bone defect 
after debridement, allograft was added to the autograft (Fig. 3D).

Graft was mixed with antibiotics based on the results of pre-operative cultures if available. If no culture result was available, vancomycin $2 \mathrm{~g}$ plus gentamicin $80 \mathrm{mg}$ was added to ensure both gram-positive and gram-negative coverage. After radical debridement, the resulting void was packed with AIBG as solidly as possible (Fig. 1C). Sites of fixation were based on bone quality and clinical condition. Pedicle screws were inserted into segments with sufficient bone mass to secure the screw and far enough away from the destroyed areas of bone to avoid recurrence of infection.

\section{Results}

Thirteen men and eight women with a mean age of 62.7 years old (34-85) underwent TIDF with AIBG. Patients were followed for a mean of 38.6 months (24-57). Although there were no infection-related deaths, one patient expired at 36 months as a result of intracranial hemorrhage. Prior to surgery, the most common presenting symptoms were back pain $(>90 \%)$ followed by bilateral leg symptoms in ten cases (47.6\%) and unilateral symptoms in four cases (19\%). Ten patients had epidural abscess and eleven had pre-existing spinal degenerative changes such as hypertrophic ligamentum flavum and degenerative disc disease. Fourteen patients $(66.7 \%)$ received parenteral antibiotics prior to surgery. The affected levels were T10-11 (2 patients), T12-L1 (2 patients), T12-L2 (1 patient), L1-2 (1 patient), L2-3 (3 patients), L3-4 (4 patients), L4-5 (3 patients), and L5-S1 (5 patients). Pedicle screws were used in most cases (19/21, 90\%). Two patients receive TIDF without screws. Nine patients received one pedicle screw above the lesion segment and one below, five patients received two above and two below, three patients received three above and three below, one patient received one above and two below, and one patient received two above and one below.

\section{Infection status}

The most common pathogens were methicillin sensitive Staphylococcus aureus (MSSA) (14.3\%) and Escherichia coli (14.3\%). One case (4.8\%) had multiple pathogens (S. aureus and Staphylococcus haemolyticus). The positive intra-operative culture rate was $52.4 \%(11 / 21)$, and total positive culture rate was $71.4 \%$ (15/21). Mean CRP improved from $56.5 \mathrm{mg} / \mathrm{L}$ pre-operatively to $33.3 \mathrm{mg} / \mathrm{L}$ at 1 week postoperatively, $24.0 \mathrm{mg} / \mathrm{L}$ at 2 weeks, $11.6 \mathrm{mg} / \mathrm{L}$ at 4 weeks, and $5.2 \mathrm{mg} / \mathrm{dl}$ at 6 wks (Fig. 2). Fourteen patients $(66.7 \%)$ started treatment with antibiotics prior to their operations (mean pre-operative use 11.3 days). Among the patients with positive cultures, the sensitivity rate of antibiotics used in the bone graft was $80 \%(12 / 15)$.

\section{Neurologic status}

Neurologic status was also evaluated. Prior to surgery, 16 patients had a neurologic status of ASIA D and five were ASIA E. Three months post-operatively, 20 patients were ASIA E. None of the patients suffered neurologic injury during the operation. 


\section{Comparison to bone graft without antibiotics}

We compared the length of time these 21 AlBG-treated patients received post-operative antibiotic treatment with that of 28 patients (controls) consecutively treated with the same TIDF procedure but without antibiotics in the bone graft in one of our previous studies [5]. As can be seen in Table 1, Table 2, and Fig. 2, both groups had stable CRP decline after surgical treatment. There was no difference in pre-op CRP levels ( 63.9 vs $56.5, p=0.669)$. Even with a shorter mean post-op IV antibiotic duration ( 21.0 days vs 39.8 days, $p<0.005)$, the AIBG group had stable decline in mean CRP level that was closer to the normal range at 6 weeks post op (CRP: $13 \mathrm{mg} / \mathrm{L}$ vs $5.2 \mathrm{mg} / \mathrm{L}, \mathrm{p}<0.05$ ). The AIBG group had a greater reduction in CRP than the control group (Tables 1 and 2).

Table 1

Comparison between TIDF with Non-AIBG and AIBG for the treatment of PDVO

\begin{tabular}{|c|c|c|c|}
\hline & $\begin{array}{l}\text { Non antibiotic-impregnated } \\
\text { (Control) }\end{array}$ & $\begin{array}{l}\text { Antibiotic-impregnated } \\
\text { (Study) }\end{array}$ & $\begin{array}{l}\mathrm{p}- \\
\text { value }\end{array}$ \\
\hline Patients (n) & 28 & 21 & - \\
\hline Age (yr) & $60.4(37-86)$ & $62.7(34-85)$ & 0.143 \\
\hline Male/Female (no.) & $13 / 15$ & $13 / 8$ & - \\
\hline $\begin{array}{l}\text { Days of post-op IV } \\
\text { antibiotics }\end{array}$ & $39.8(28-84)$ & $21.0(7-34)$ & $<.005^{\star}$ \\
\hline CRP (pre-op) & 63.9 mg/L (3-197) & $56.5 \mathrm{mg} / \mathrm{L}(2.9 \sim 145.6)$ & 0.669 \\
\hline CRP (1 week post-op) & $44.7(2.0-98.0)$ & $33.3(1.6-109.7)$ & 0.121 \\
\hline CRP (2 weeks post-op) & $32.7(1.0-172)$ & $24.0(1.4-163.0)$ & 0.094 \\
\hline CRP (4 weeks post-op) & $20.8(3.0-99.0)$ & $11.6(1.3-93.6)$ & 0.061 \\
\hline CRP (6 weeks post-op) & $13.0(1.0-62)$ & $5.2(0.4-27.4)$ & $0.010 *$ \\
\hline$\triangle \mathrm{VAS}$ back pain & $8.3 \diamond 2.4$ & $7.2 \diamond 2.3$ & \\
\hline kyphotic angle correction & $9.7^{\circ}$ & $7.9^{\circ}$ & \\
\hline \multicolumn{4}{|c|}{ TIDF: Transforaminal interbody debridement and fusion } \\
\hline \multicolumn{4}{|c|}{ AIBG: Antibiotics impregnated bone graft } \\
\hline \multicolumn{4}{|c|}{ PDVO: pyogenic discitis and vertebral osteomyelitis } \\
\hline \multicolumn{4}{|l|}{ Mann-Whitney U test, Mean } \\
\hline${ }^{*} p<0.05$ was considered $s$ & tistically significant & & \\
\hline
\end{tabular}


Table 2

Difference in CRP reduction between Non-AIBG and AIBG for the treatment of PDVO

\begin{tabular}{|c|c|c|}
\hline & $\begin{array}{l}\text { Non antibiotic-impregnated } \\
\text { (Control) }\end{array}$ & $\begin{array}{l}\text { Antibiotic-impregnated } \\
\text { (Study) }\end{array}$ \\
\hline CRP Reduction (post-op $1 \mathrm{wk}$ ) & $32 \%$ & $41 \%$ \\
\hline $\begin{array}{l}\text { CRP Reduction (post-op } 2 \\
\text { wks) }\end{array}$ & $49 \%$ & $59 \%$ \\
\hline $\begin{array}{l}\text { CRP Reduction (post-op } 4 \\
\text { wks) }\end{array}$ & $66 \%$ & $79 \%$ \\
\hline $\begin{array}{l}\text { CRP Reduction (post-op } 6 \\
\text { wks) }\end{array}$ & $80 \%$ & $91 \%$ \\
\hline \multicolumn{3}{|c|}{$* \%$ Reduction in CRP $=\frac{(\text { post }- \text { op CRP })-(\text { pre }- \text { op CRP })}{\text { pre-op CRP }} \times 100 \%$} \\
\hline \multicolumn{3}{|c|}{ TIDF: Transforaminal interbody debridement and fusion } \\
\hline \multicolumn{3}{|c|}{ AIBG: Antibiotics impregnated bone graft } \\
\hline PDVO: pyogenic discitis and $v \epsilon$ & ebral osteomyelitis & \\
\hline
\end{tabular}

\section{Fusion status and sagittal correction}

Fusion status was evaluated at last follow-up. Solid unions were found in seventeen (17/21) patients (81\%), pseudarthrosis in two $(2 / 21,9.5 \%)$, and inadequate union in two $(2 / 21,9.5 \%)$. Ten cases had improvement in the lordotic over the infected levels after surgery. Mean kyphotic angle pre-opearatively was $13.6 \pm 7.2^{\circ}$. It improved to $1.5 . \pm 5.5^{\circ}(P<0.001)$ immediately post-operatively and $5.7 . \pm 10.1^{\circ}(P<$ 0.05 ) at final follow-up for a mean kyphotic angle correction of $7.9^{\circ}$ at final follow-up.

\section{Functional Outcome}

Mean back pain VAS improved from $7.2 \pm 0.9$ (range: $4.5-8.8$ ) pre-operatively to $2.3 \pm 1.3$

(range: $0.7-5.5$ ) at one-month follow-up. Two patients complained of donor site pain at initial follow-up, which was relieved at final follow-up.

Kirkaldy-Willis functional outcome at the last follow-up showed excellent results in 8 cases; good results in 9 cases; fair results in 3 cases; and poor results in 1 patient who underwent revision surgery due to a deep wound infection.

\section{Revisions}

Two patients had revision surgery. One was due to pseudarthrosis with broken S1 screws one year postoperatively. Revision posterolateral fusion performed was with allograft and demineralized bone matrix (DBM). Subsequent standing X-rays showed union with clinical back pain relief. The other patient 
developed a deep wound infection one-month post-TIDF. Anterior debridement and corpectomy with strut allograft reconstruction was performed for this patient.

\section{Discussion}

Parenteral antibiotic treatment is the primary treatment for PDVO; however, surgical debridement may be indicated in patients when conservative treament fails. Some have suggested that infections can be better controlled if the patient receives early surgical debridement with parenteral antibiotics [24, 25]. The previous gold-standard surgical approach for PDVO was the anterior approach. Although this approach may enable better debridement, it is accompanied by a higher morbidity rate than the posterior approach $[26,27]$. Thus, the posterior-only approach has recently become more popular. It is performed with equivalent infection control but with lower surgical risk than the anterior approach $[13,28]$. Earlier studies of posterior lumbar interbody fusion (PLIF) with instrumentation reported good infection control $[13,28]$. PLIF requires a laminectomy and retraction of the nerve roots, which may injure the nerve and result in spinal instability. TIDF plus pedicle screw fixation was thus invented to treat PDVO [5]. This method prevents postoperative instability and decreases the risk of nerve injury. Previous studies have reported promising results with the use of TIDF combined with posterior instrumentation to treat either nonpyogenic or pyogenic lumbar spine infection $[5,16]$. This procedure offers immediate stability to prevent further kyphotic change and accelerates infection control. As a result, patients benefit from decreased back pain, decreased use of pain medicine, and improved quality of life $[5,16]$.

\section{Parenteral antibiotic treatment in PDVO}

Although TIDF surgery can remove infected disc, endplate, and sequestrum in the treatment of PDVO, post operative antibiotics are always required. There is no consensus as regarding the period of intravenous antibiotics, and treatment is mainly based on personal experience or expert opinion rather than evidence-based recommendations. Bernard et al. suggested that the standard duration of antibiotics treatment for pyogenic vertebral osteomyelitis could be reduced to six weeks in a multicenter noninferiority trial[29]; this reduction in antibiotic treatment period also decreases the risk of side effects, economic burden, and development of antibiotic resistance [8, 30]. Park et al. further considered the optimal duration of antibiotics therapy based on the patient's risk of recurrence [31]. In that study, 314 patients were evaluated for recurrence and multivariate analysis revealed methicillin-resistant $S$. aureus (MRSA) infection, undrained paravertebral/psoas abscesses, and end-stage renal disease to be independent baseline risk factors for recurrence. They concluded that a prolonged duration ( $\geq 8$ weeks) of antibiotics therapy should be used in this high-risk group. In this study, no patients met these criteria. Thus, duration of post-operative antibiotics treatment was decided on based on relief of clinical symptoms and improvement in lab values. Several previous studies suggest post-operative antibiotic treatment for as short as four weeks can be effective $[5,16]$. In the current study, mean post-operative IV antibiotics duration was only 21 days. (Table 1 ).

\section{Antibiotic impregnated bone graft (AIBG)}


AIBG has been widely used in revision arthroplasty and trauma surgery with promising results $[17,20,21$, 32]. Biofilm-embedded pathogens require an MIC of up to 1000 -fold for elimination. Only local application of antibiotics can achieve the high concentrations required to eliminate the biofilm [33]. Although antitiotic impregnated Polymethylmethacrylate (PMMA) cement is frequently used as a temporary spacer when treating infected arthroplasty, it was not used in these cases. Borkhuu et al. reported a significant decrease in infection rate after spinal surgery in children with scoliosis due to cerebral palsy after local use of AIBG (4\%) as compared with a group in which plain bone graft was inserted (15\%) [34]. To best of our knowledge, our study is the first to employ AIBG with TIDF to treat PDVO. Antibiotics added to the bone graft in this study were based on pre-operative culture results. If the patient had no pre-operative culture result, vancomycin $2 \mathrm{~g}$ combined with gentamicin $80 \mathrm{mg}$ were used to cover both gram-positive and gram-negative bacteria. Vancomycin was chosen because it is has been found to be efficacious against gram-positive cocci, the most common cause of PDVO [2]. Gentamycin was chosen because its synergic effect with vancomycin against MSSA $[35,36]$. Another advantage of vancomycin and gentamicin is better penetration and uptake into the intervertebral disc because they are positively charged [37, 38].

\section{Dose of antibiotic in bone graft}

There is no consensus on the optimal dose for local antibiotic use [17]. Some concerns about inhibition of osteogenesis by antibiotics have been raised [39], though no significant difference in the osteogenic activity was noted in one animal study [40]. We used the same dose that a previous study used in onestage revision arthroplasty, $100 \mathrm{mg}$ vancomycin per $1 \mathrm{cc}$ of bone[21]. In that article, the author mentioned that high tissue and low serum levels of vancomycin could be due to its poor tissue penetration. Although very high concentrations of antibiotics were used, no significant adverse effects were noted. Seber et al. measured gentamicin levels in urine after insertion of gentamicin-loaded xenograft in the treatment of osteomyelitis and found the mean level at $24 \mathrm{~h}$ to be $4 \mu \mathrm{g} / \mathrm{mL}$, falling below the effective level of $0.5 \mu \mathrm{g} / \mathrm{mL}$ after 8 days. [41] They found no cases of nephrotoxicity or ototoxicity. We found no significant change in renal function during follow-up.

\section{Conclusion}

Based on our study, the technique of TIDF with AIBG is an effective and safe technique in the treatment of pyogenic discitis and vertebral osteomyelitis. This technique can be used to achieve superior local infection control as evidenced by faster normalization of CRP levels, possibly shorten the duration of post-operative IV antibiotic use, correct the kyphotic angle, and achieve satisfactory outcomes.

\section{Abbreviations}

PDVO

pyogenic discitis and vertebral osteomyelitis

TIDF

transforaminal interbody debridement and fusion 
AIBG

antibiotic-impregnated bone graft

VAS

visual analog scale

CRP

C-reactive protein

ASIA

American Spinal Injury Association

\section{Declarations}

\section{Ethics approval and consent to participate}

The study was approved by the Chang Gung Medical Foundation Institutional Review Board. The IRB No.: $201801075 B 0$.

\section{Consent for publication}

The IRB (Chang Gung Medical Foundation Institutional Review Board) reviewed and determined that the need for consent was deemed unnecessary according to the retrospective case research, cases treated or diagnosed by clinical routines. The IRB is organized and operates in accordance with Good Clinical Practice and the applicable laws and regulations.

\section{Availability of data and materials}

All data generated or analyzed during this study are included in this published article.

\section{Competing interests}

The authors declare that they have no competing interests

\section{Funding}

There is no funding support in this study.

\section{Authors' contributions}

HKC: Writing-Original Draft

AW: Writing-Review \& Editing 
MLL: Methodology, Investigation

$\mathrm{CCH}$ : Resources, Methodology

RWW: Resources, Investigation

SHC: Resources, Conceptualization

HTC: Methodology, Investigation

TCY: Conceptualization, Supervision, Resources

All authors have read and approved the manuscript

\section{Acknowledgements}

Not applicable

\section{References}

1. Hadjipavlou AG, Mader JT, Necessary JT, Muffoletto AJ: Hematogenous pyogenic spinal infections and their surgical management. Spine (Phila Pa 1976), 2000 25(13): p 1668-79 2000.

2. Gouliouris T, Aliyu SH, Brown NM. Spondylodiscitis: update on diagnosis and management. J Antimicrob Chemother. 2010;65(Suppl 3):iii11-24. 10.1093/jac/dkq303.

3. Abramovitz JN, Batson RA, Yablon JS. Vertebral osteomyelitis. The surgical management of neurologic complications. Spine. 1986;11(5):418-20.

4. Arnold PM, Baek PN, Bernardi RJ, Luck EA, Larson SJ. Surgical management of nontuberculous thoracic and lumbar vertebral osteomyelitis: report of 33 cases. Surgical neurology. 1997;47(6):55161.

5. Lu ML, Niu CC, Tsai TT, Fu TS, Chen LH, Chen WJ. Transforaminal lumbar interbody debridement and fusion for the treatment of infective spondylodiscitis in the lumbar spine. Eur Spine J. 2015;24(3):555-60. 10.1007/s00586-014-3585-3.

6. Osenbach RK, Hitchon PW, Menezes AH. Diagnosis and management of pyogenic vertebral osteomyelitis in adults. Surgical neurology. 1990;33(4):266-75.

7. Eismont FJ, Bohlman HH, Soni PL, Goldberg VM, Freehafer AA. Pyogenic and fungal vertebral osteomyelitis with paralysis. The Journal of bone joint surgery American volume. 1983;65(1):19-29.

8. Emery SE, Chan DP, Woodward HR. Treatment of hematogenous pyogenic vertebral osteomyelitis with anterior debridement and primary bone grafting. Spine. 1989;14(3):284-91.

9. Hodgson AR, Stock FE, Fang HS, Ong GB. Anterior spinal fusion. The operative approach and pathological findings in 412 patients with Pott's disease of the spine. Br J Surg. 1960;48:172-8. 
10. Kemp HB, Jackson JW, Jeremiah JD, Cook J. Anterior fusion of the spine for infective lesions in adults. The Journal of bone joint surgery British volume. 1973;55(4):715-34.

11. Fritzell $P$, et al.: Chronic low back pain and fusion: a comparison of three surgical techniques: a prospective multicenter randomized study from the Swedish lumbar spine study group. Spine (Phila Pa 1976), 2002 27(11): $p$ 1131-41.

12. Sasso RC, et al. Analysis of operative complications in a series of 471 anterior lumbar interbody fusion procedures. Spine (Phila Pa 1976). 2005;30(6):p. 670-4.

13. Lee JS, Suh KT. Posterior lumbar interbody fusion with an autogenous iliac crest bone graft in the treatment of pyogenic spondylodiscitis. The Journal of bone joint surgery British volume. 2006;88(6):765-70. 10.1302/0301-620X.88B6.17270.

14. Przybylski GJ, Sharan AD. Single-stage autogenous bone grafting and internal fixation in the surgical management of pyogenic discitis and vertebral osteomyelitis. Journal of neurosurgery. 2001;94(1 Suppl):1-7.

15. Rath SA, Neff U, Schneider O, Richter HP. Neurosurgical management of thoracic and lumbar vertebral osteomyelitis and discitis in adults: a review of 43 consecutive surgically treated patients. Neurosurgery. 1996;38(5):926-33.

16. Chang CW, Tsai TT, Niu CC, Fu TS, Lai PL, Chen LH, Chen WJ. Transforaminal Interbody Debridement and Fusion to Manage Postdiscectomy Discitis in Lumbar Spine. World Neurosurg. 2019;121:e75560. 10.1016/j.wneu.2018.09.211.

17. Anagnostakos K, Schroder K. Antibiotic-impregnated bone grafts in orthopaedic and trauma surgery: a systematic review of the literature. Int J Biomater. 2012;2012:538061. 10.1155/2012/538061.

18. Chan YS, Ueng SW, Wang CJ, Lee SS, Chen CY, Shin CH. Antibiotic-impregnated autogenic cancellous bone grafting is an effective and safe method for the management of small infected tibial defects: a comparison study. J Trauma. 2000;48(2):246-55.

19. Winkler H, Kaudela K, Stoiber A, Menschik F. Bone grafts impregnated with antibiotics as a tool for treating infected implants in orthopedic surgery - one stage revision results. Cell Tissue Banking 2006.

20. Buttaro M, Comba F, Piccaluga F. Vancomycin-supplemented cancellous bone allografts in hip revision surgery. Clin Orthop Relat Res. 2007;461:74-80. 10.1097/BLO.0b013e318073c290.

21. Buttaro MA, Pusso R, Piccaluga F. Vancomycin-supplemented impacted bone allografts in infected hip arthroplasty. Two-stage revision results. The Journal of bone joint surgery British. volume 2005;87(3):314-9.

22. Fleege $C$, Wichelhaus TA, Rauschmann M. Systemic and local antibiotic therapy of conservative and operative treatment of spondylodiscitis. Der Orthopade. 2012;41(9):727-35. 10.1007/s00132-0121920-0.

23. Bernard TN Jr, Kirkaldy-Willis WH. Recognizing specific characteristics of nonspecific low back pain. Clin Orthop Relat Res 1987(217):266-280. 
24. Sheha AF. Surgical Management of Post-Discectomy Spondylodiscitis with Transforaminal Lumbar Interbody Fusion (TLIF) and Posterior Instrumentation. Life Science Journal. 2011;8(4):296-303.

25. Hamdan TA. Postoperative disc space infection after discectomy: A report on thirty-five patients. Int Orthop 2012 Feb; 36(2): 445-450.

26. Sasso RC, Best NM, Mummaneni PV, Reilly TM, Hussain SM. Analysis of operative complications in a series of 471 anterior lumbar interbody fusion procedures. Spine. 2005;30(6):670-4.

27. Fritzell P, Hagg O, Wessberg P, Nordwall A, Swedish Lumbar Spine Study G. Chronic low back pain and fusion: a comparison of three surgical techniques: a prospective multicenter randomized study from the Swedish lumbar spine study group. Spine. 2002;27(11):1131-41.

28. Endres S, Wilke A. Posterior interbody grafting and instrumentation for spondylodiscitis. J Orthop Surg (Hong Kong). 2012;20(1):1-6. 10.1177/230949901202000101.

29. Bernard L, Dinh A, Ghout I, Simo D, Zeller V, Issartel B, Le Moing V, Belmatoug N, Lesprit P, Bru J-P, et al. Antibiotic treatment for 6 weeks versus 12 weeks in patients with pyogenic vertebral osteomyelitis: an open-label, non-inferiority, randomised, controlled trial. The Lancet. 2015;385(9971):875-82. 10.1016/s0140-6736(14)61233-2.

30. Rice LB. The Maxwell Finland Lecture: for the duration-rational antibiotic administration in an era of antimicrobial resistance and clostridium difficile. Clin Infect Dis. 2008;46(4):491-6. $10.1086 / 526535$.

31. Park KH, Cho OH, Lee JH, Park JS, Ryu KN, Park SY, Lee YM, Chong YP, Kim SH, Lee SO, et al. Optimal Duration of Antibiotic Therapy in Patients With Hematogenous Vertebral Osteomyelitis at Low Risk and High Risk of Recurrence. Clin Infect Dis. 2016;62(10):1262-9. 10.1093/cid/ciw098.

32. Van Vugt TA, Geurts J, Arts JJ. Clinical Application of Antimicrobial Bone Graft Substitute in Osteomyelitis Treatment: A Systematic Review of Different Bone Graft Substitutes Available in Clinical Treatment of Osteomyelitis. Biomed Res Int. 2016;2016:6984656. 10.1155/2016/6984656.

33. Saginur R, Stdenis M, Ferris W, Aaron SD, Chan F, Lee C, Ramotar K. Multiple combination bactericidal testing of staphylococcal biofilms from implant-associated infections. Antimicrob Agents Chemother. 2006;50(1):55-61. 10.1128/AAC.50.1.55-61.2006.

34. Borkhuu B, Borowski A, Shah SA, Littleton AG, Dabney KW, Miller F. Antibiotic-loaded allograft decreases the rate of acute deep wound infection after spinal fusion in cerebral palsy. Spine. 2008;33(21):2300-4. 10.1097/BRS.0b013e31818786ff.

35. Houlihan HH, Mercier RC, Rybak MJ. Pharmacodynamics of vancomycin alone and in combination with gentamicin at various dosing intervals against methicillin-resistant Staphylococcus aureusinfected fibrin-platelet clots in an in vitro infection model. Antimicrob Agents Chemother. 1997;41(11):2497-501.

36. Tsuji BT, Rybak MJ. Short-course gentamicin in combination with daptomycin or vancomycin against Staphylococcus aureus in an in vitro pharmacodynamic model with simulated endocardial vegetations. Antimicrob Agents Chemother. 2005;49(7):2735-45. 10.1128/AAC.49.7.27352745.2005 . 
37. Riley LH 3rd, Banovac K, Martinez OV, Eismont FJ. Tissue distribution of antibiotics in the intervertebral disc. Spine. 1994;19(23):2619-25.

38. Zhu Q, Gao X, Li N, Gu W, Eismont F, Brown MD. Kinetics of charged antibiotic penetration into human intervertebral discs: A numerical study. J Biomech. 2016;49(13):3079-84.

10.1016/j.jbiomech.2016.07.012.

39. Gray JC, Elves MW. Osteogenesis in bone grafts after short-term storage and topical antibiotic treatment. An experimental study in rats. The Journal of bone joint surgery British. volume 1981;63$\mathrm{B}(3): 441-5$.

40. Petri WH 3. Osteogenic activity of antibiotic-supplemented bone allografts in the guinea pig. $\mathrm{J}$ Oral Maxillofac Surg. 1984;42(10):631-6. rd. .

41. Seber S, Gunal I, Gokturk E. Antibiotic-impregnated xenografts in the treatment of chronic osteomyelitic cavities. Seven cases followed for 3 to 5 years. Int Orthop. 1998;22(3):197-9.

\section{Figures}
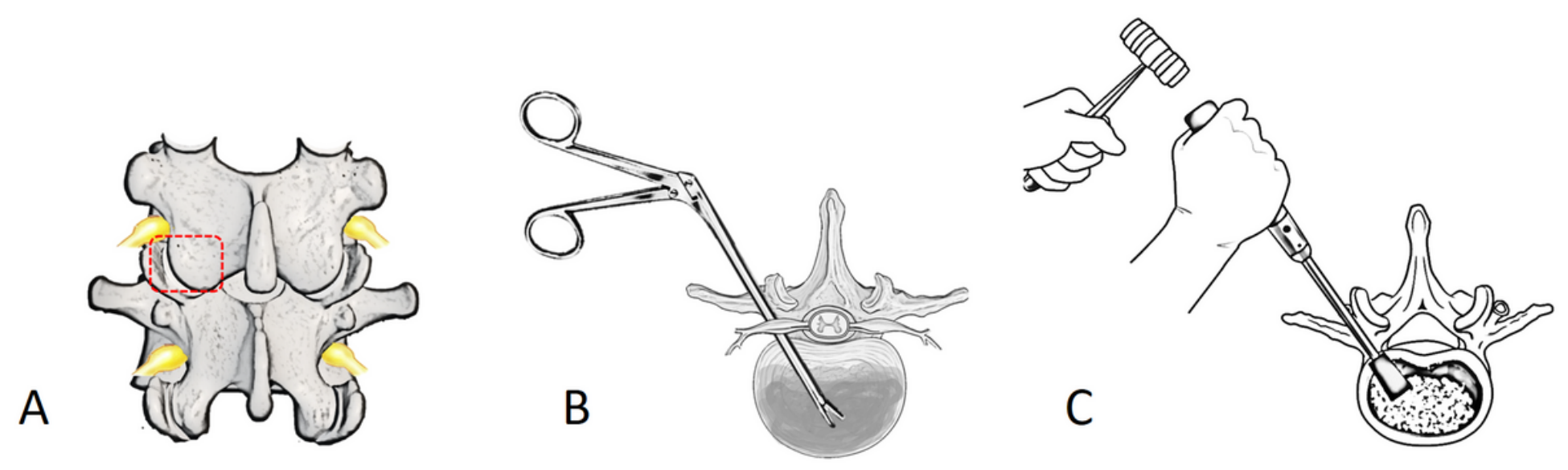

Figure 1

Fig.1A: The area of bony removal and route of access to the intervertebral space. Fig.1B: Transformainal radical debridement to remove abscess, infected disc material, endplates, and sequestrum from the intervertebral space by curved curette and disc roungeur. Fig.1C The intervertebral space was impacted with AIBG as solid as possible. 


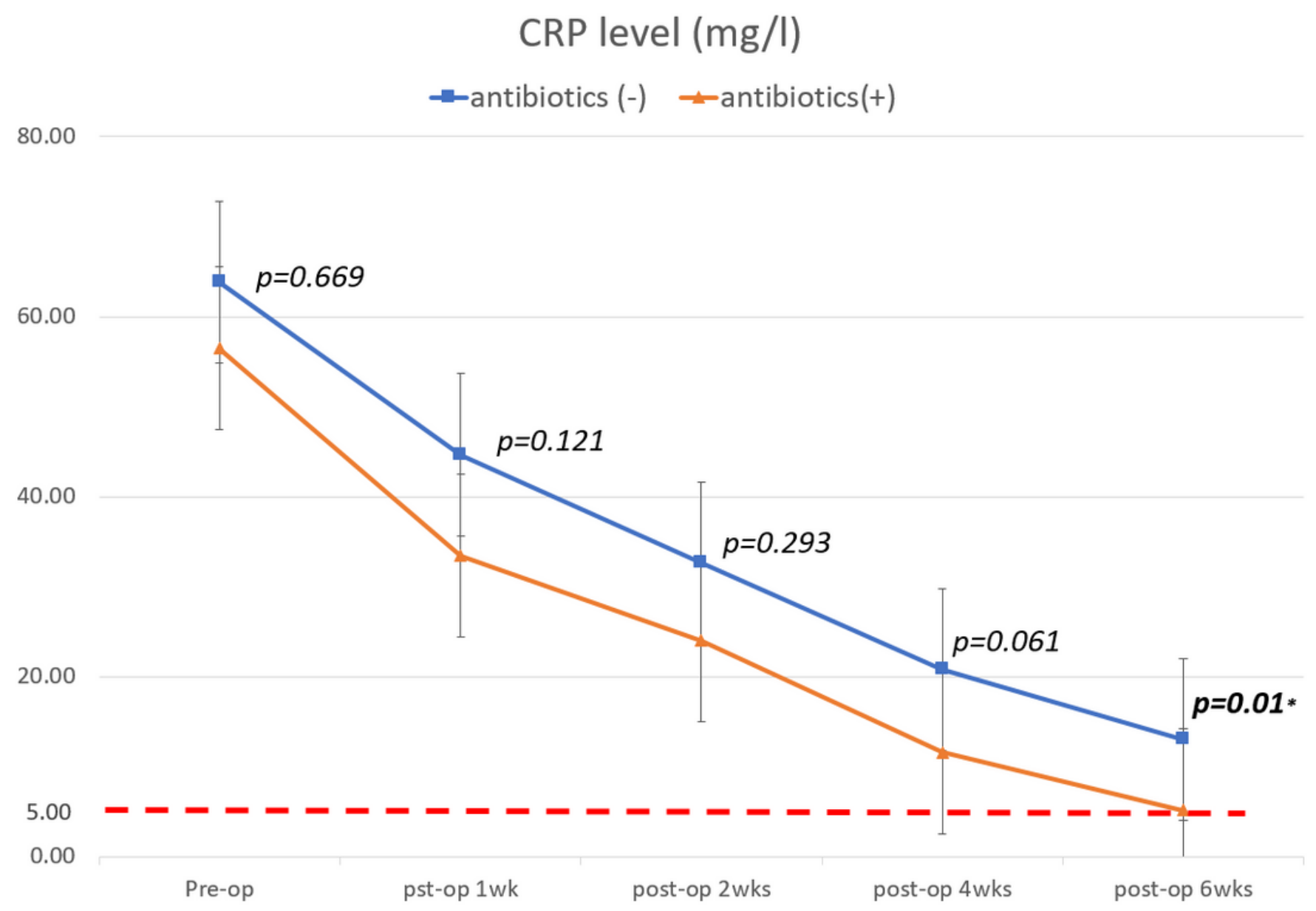

Figure 2

Compared the same TIDF procedure with and without antibiotics impregnated bone grafts. Even with a shorter mean post-op IV antibiotic duration (21.0 days vs 39.8 days), the AIBG group had stable decline in mean CRP level that was closer to the normal range at 6 weeks postoperatively. (CRP: $13 \mathrm{mg} / \mathrm{L}$ vs 5.2 $\mathrm{mg} / \mathrm{L}, \mathrm{p}<0.05)$ 


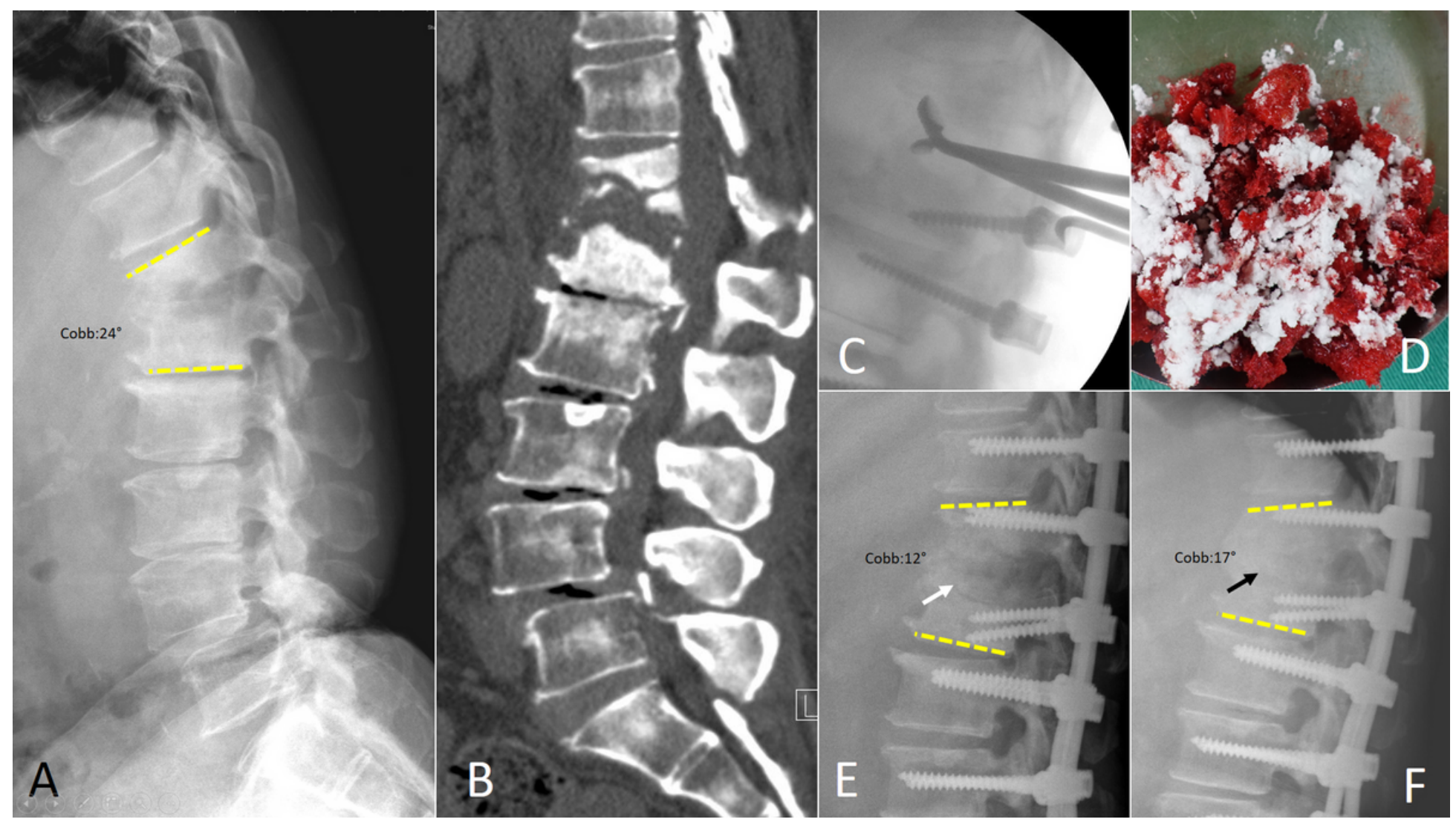

Figure 3

A $54 \mathrm{y} /$ o female woman with PDVO at T12/L1 level had a pre-operative CRP level of $29.4 \mathrm{mg} / \mathrm{L}$. She also had multiple lumbar degenerative disc disease and degenerative spondylolisthesis with lumbar spinal stenosis at L45 area. Figure 3A: L-spine XR and spinal CT showed T12/L1 PDVO with endplate destruction and the pre-op Cobb angle was $24^{\circ}$. Figure $1 \mathrm{C}$ : Necrotic tissue and debris were currated and removed through transforaminal route at T12/L1 level. Figure 1D: Auto-iliac bone graft combined allograft was mixed homogeneously with antibiotics. Figure 1E: Bone graft was firmly impacted into the disc level (white arrow) and the post-op 1 month Cobb angle was 12 degree. Patient received 2 weeks of post-surgery IV antibiotics and the CRP level declined to $7.2 \mathrm{mg} / \mathrm{L}$,then the patient was transitioned to oral antibiotics. Figure 1F: Post-op 2 years showed solid fusion at T12/L1 level (black arrow) and the residual Cobb angle was $17^{\circ}$. 


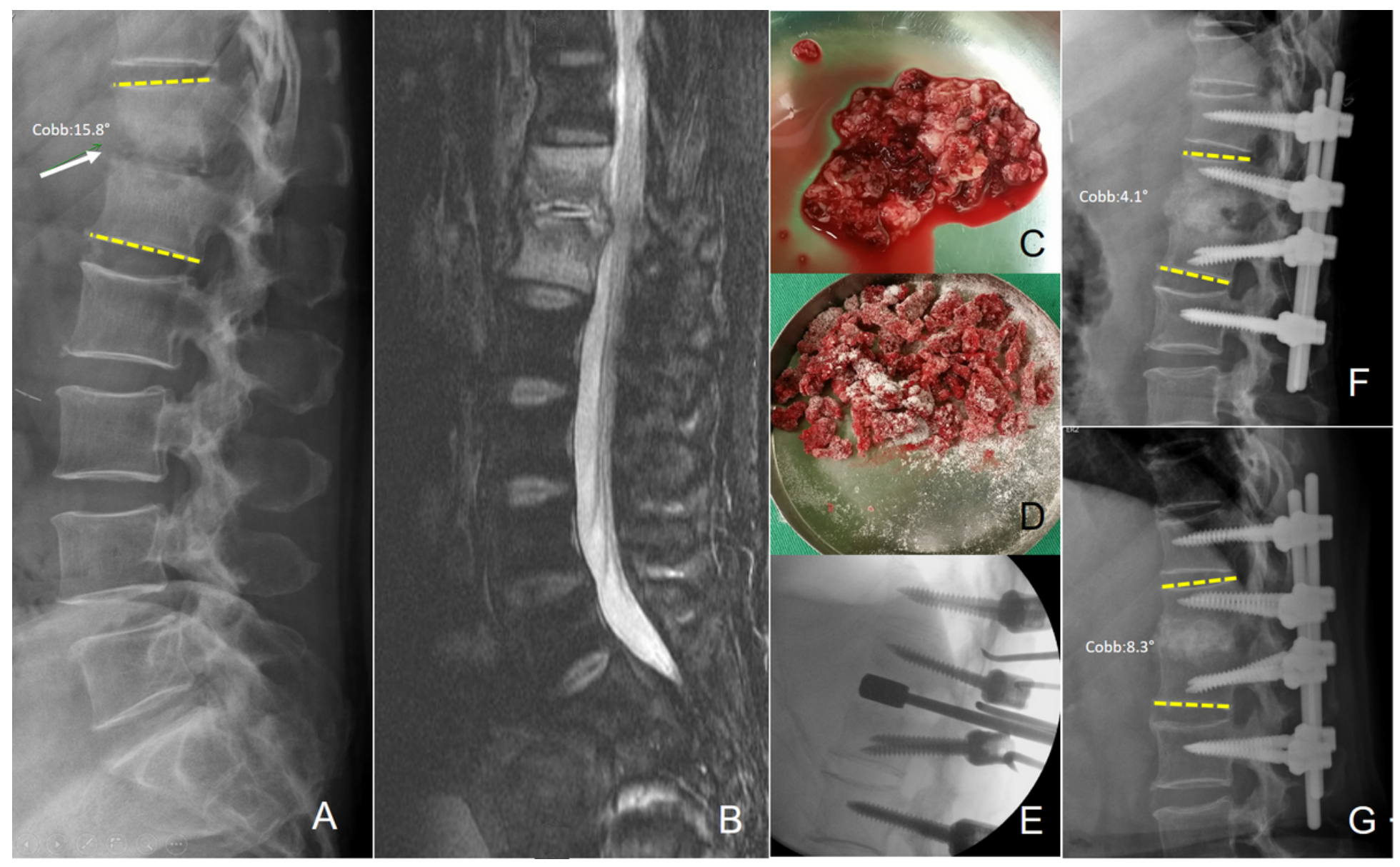

Figure 4

A 60-year-old woman with PDVO at T12/L1 level had a pre-operative CRP level of $60.7 \mathrm{mg} / \mathrm{L}$. Figure 4A-B: L-spine XR and MRI showed T12/L1 PDVO with endplate destruction and the pre-op Cobb angle was $15.8^{\circ}$. Figure 4C: Necrotic tissue and debris were removed after transforaminal T12/L1 interbody debridement. Figure 4D: Auto-iliac bone graft was mixed homogeneously with antibiotics. Figure 4E: Bone graft was firmly impacted into the disc level. Patient received 1 week of post-surgery IV antibiotics, the patient was transitioned to oral antibiotics, and CRP level continued to decline to $1.3 \mathrm{mg} / \mathrm{L}$ the following week. Figure 4F: Post-op immediately showed the initial kyphotic Cobb angle was corrected to $4.1^{\circ}$. Figure 4G: A solid T12/L1 interbody fusion was observed 1 year postoperatively and the residual Cobb angle was $8.3^{\circ}$ 